\title{
Padrões de aleitamento materno exclusivo e internação por diarréia entre 1999 e 2008 em capitais brasileiras
}

\author{
Exclusive breastfeeding and diarrhea hospitalization patterns \\ between 1999 and 2008 in Brazilian State Capitals
}

\author{
Cristiano Siqueira Boccolini ${ }^{1}$ \\ Patricia de M oraes M ello Boccolini ${ }^{2}$ \\ Márcia Lazaro de Carvalho ${ }^{3}$ \\ $M$ aria Inês Couto de Oliveira ${ }^{4}$
}

${ }^{1}$ Escola Nacional de Saúde Pública, Fundação O swaldo Cruz. Rua Leopoldo Bulhões, 1480/806, M anguinhos. 21041210 Rio deJaneiro RJ. cristianoboccolini@gmail.com ${ }^{2}$ Instituto de Estudos em Saúde Coletiva, UniversidadeFederal do Rio deJaneiro.

${ }^{3}$ Departamento de Epidemiologia e M étodos Quantitativosem Saúde, Escola Nacional de Saúde Pública, Fundação O swaldo Cruz.

${ }^{4}$ Departamento de Epidemiologia e Bioestatística, Instituto de Saúdeda Comunidade, UniversidadeFederal Fluminense.
Abstract The prevalence of breastfeeding hasincreased over the past two decades in Brazil, as a result of public breastfeeding policies. The scope of this paper is to analyze the correlation between theincreasein the prevalence of breastfeeding and hospitalization rates due to diarrhea. It is an epidemiological ecological study, based on secondary data from Brazilian Capital Cities and the Federal District. The prevalence of breastfeeding, the number of live births, and cases of hospitalization due to diarrhea were compared for the years 1999 and 2008 and the Spearman non-parametric test was used to correlate the variables. During the period, 1,329,618 children under one year of age in 1999 and 2008 were studied. The increase in the prevalence of exclusive breastfeeding among children under 4 months old had a negative correlation with hospitalization rates dueto diarrhea ( $R h o=-0.483, p=0.014$ ). This correlation was stronger for girls ( $R$ ho $=-0.521$, $p=0.008$ ) than for boys ( $R h 0=-0.476, p=0.016)$. The increase in the prevalence of breastfeeding between 1999 and 2008 appears to be correlated to a reduction in hospitalization rates due to diarrhea over the same period, corroborating the importance of public policies to protect, support and promote breastfeeding.

Key words Maternal and child health, Breastfeeding, Breastfed infants, H ospitalization, Infantile diarrhea, Live births
Resumo A prevalência do aleitamento materno tem aumentado nas duas últimas décadas como resultado das políticas públicas deincentivo a esta prática. Trata-se de estudo epidemiológico ecológico, com base em dados secundários das Capitais Brasileiras e Distrito Federal. As prevalências de aleitamento materno, a população de nascidos vivos e os casos de internação hospitalar por diarreias foram comparados entre os anos de 1999 e 2008. Foi utilizado o teste não-paramétrico de Spearman para correlacionar as variáveis. Foram estudados 1.329.618 nascidos vivos no período. 0 aumento da prevalência dealeitamento materno exclusivo em crianças com menos de 4 meses de vida teve correlação negativa com as taxas deinternação por diarreias $(r=-0,483, p=0,014)$, sendo essa correlação mais forte para meninas ( $r$ $=-0,521, p=0,016)$ que para os meninos $(r=$ $0,476, p=0,008)$. 0 aumento da prevalência de al eitamento materno exclusivo entre 1999 e 2008 parece estar correlacionado com a diminui ção das taxas de internação hospitalar por diarreias no mesmo período, corroborando a importância das políticas públicas de promoção, proteção e apoio do aleitamento materno.

Palavras-chave Saúde materno-infantil, Aleitamento materno, Lactente, H ospitalização, Diarreia infantil, Nascidos vivos 


\section{Introdução}

Até dois terços das mortes infantis evitáveis podem ser atribuídas às diarreias ${ }^{1}$, as quais podem ser responsáveis por cerca de quatro milhões de mortes por ano nos países em desenvolvimento².

Apesar da incidência da hospitalização por doenças diarreicas apresentar tendência a diminuição $0^{3,4}$, a morbidade destas corresponde a segunda causa de internações em crianças com menos de um ano no Estado do Rio de Janeiro ${ }^{5}$, e 0 aleitamento materno exclusivo pode reduzir em $53 \%$ a incidência dessas hospitalizações ${ }^{6}$.

Por outro lado, na última década verificou-se o aumento da prevalência de al eitamento materno exclusivo em menores de quatro meses de $35,5 \%$ (1999) para 51,2\% (2008) nas capitais brasileiras eno Distrito Federal ${ }^{7}$, o que podeter contribuído para a redução da morbimortalidade infantil ${ }^{1,8-10}$ e para a diminuição da incidência de doenças diarreicas ${ }^{11-13}$.

A redução da mortalidade infantil no Brasil de 1980 se deu sem mudanças significantes de renda familiar, mas o sistema de saúde brasileiro passou a adotar políticas importantes de atenção à saúde infantil, incluindo a promoção do aleitamento materno. Essa ten dência prosseguiu nas décadas seguintes, principalmente devido aos fenômenos de aumento da urbanização e de redução da fertilidade e a expansão das políticas de saúde pública ${ }^{14}$.

0 principal mecanismo fisiológico para a redução da morbimortalidade conferido pelo leite materno são seus compostos imunológicos ${ }^{15,16}$, como a I gA-secretória ${ }^{17,18}$ eos oligossacarídeos ${ }^{19,20}$ que se adaptam às necessidades de cada criança.

Este estudo teve como objetivo verificar se 0 incremento na prevalência do aleitamento materno observado nas capitais brasi leiras eno Distrito Federal entre 1999 e 2008 esteve correlacionado com a redução das taxas de internação hospitalar por diarreias entre as crianças com menos de um ano de vida no período.

\section{Metodologia}

Trata-sedeestudo epidemiológico ecológico, cuja população estudada foi a de crianças menores de um ano residentes nas capitais brasileiras e Distrito Federal nos anos de 1999 e 2008 cujos dados secundários foram agregados por município.

A população denascidos vivosem 1999 e2008 foi obtida do Sistema de Nascidos Vivos do Datasus ${ }^{21}$, por capital federal eDistrito Federal, por local deresidência, e foi utilizada como uma estimativa aproximada das crianças com menos de um ano de vida para os referentes anos.

Oscasos deinternações hospitalares em crianças com menos de um ano de vida (por local de residência) por doenças diarreicas e gastroenteritesinfecciosas de origem presumível em 1999 e em 2008 foram obtidos do Sistema de Informações H ospitalares (SIH) de acordo com a Classificação Internacional de Doenças (CID-10), em crianças com menos de um ano de vida (por local de residência). Tendo como menor nível de agregação o indivíduo, sem a possibilidade de identificação do sujeito ${ }^{21}$.

As taxas de internação por doença diarreica foram padronizadas por 1000 crianças residentes com menos de um ano de vida, tendo como base as populações obtidas do Datasus. Além disso, as taxas de internação por diarreia foram calculadas tendo como parâmetro o total de internações. As razões detaxa deinternação hospitalar por sexo foram obtidas ao se dividir a taxa de internação hospitalar dos meninos pela taxa das meninas.

Asinformações referentes ao al eitamento materno exclusivo entre crianças com menos dequatro meses devida eentrecrianças com novea doze meses de vida foram obtidas da "II Pesquisa de Prevalência de Aleitamento $M$ aterno nas capitais Brasileiras eD istrito Federal" publicada em $2009 \mathrm{e}$ com informações sobre a prevalência de aleitamento materno em 1999 e 2008. Foram utilizadas as médias pontuais da prevalência de aleitamento materno exclusivo eda prevalência dealeitamento materno em crianças com nove a doze meses incompletos devida em cada cidadereferentesà 1999 e 20087. Além disso, foi calculada a diferença de prevalência entre os dois anos, subtraindo a prevalência de2008 da prevalência de 1999.

A capital do Acre (Rio Branco) não foi incluída por não possuir informações sobre o desfecho em 200821, e a cidade do Rio de Janeiro foi excluída da análise por não ter realizado o inqué rito do M inistério da Saúdeem 19997. Além disso, somente havia dados sobre al eitamento materno em crianças de nove a doze meses de vida para os anos de 1999 e $2008 \mathrm{em} 18$ das 27 capitais brasilei ras e Distrito Federal.

Foi realizada análise univariada com o teste não paramétrico de Wilcoxon para avaliar se houve alterações estatisticamente significativas das taxas de internação hospitalar e das razões de taxas entre os anos de 1999 e 2008.

Em seguida foi estimado o coeficiente decorrelação de Spearman entre as diferenças de pre- 
valência de aleitamento materno ocorridas entre 1999 e 2008 e as diferenças de taxa de internação hospitalar ocorrida no mesmo período.

A próxima etapa da análise consistiu em classificar cada cidade em tercis de aumento das prevalências de aleitamento materno exclusivo em crianças com menos dequatro meses devida entre 1999 e 2008: o primeiro tercil foi composto pelas cidades em que se observaram os menores aumentos das prevalências (tercil dereferência), eo terceiro tercil composto pelas cidades com maiores aumentos.

A redução absoluta da taxa de hospitalizações por diarreia foi calculada subtraindo-se as taxas de 2008 das taxas de 1999, para, em seguida, serem calculadas as reduções percentuais das taxas, tendo como numeradores as reduções absolutas das taxas entre 1999 e 2008, e como denominadores as taxas de 1999.

As razões de redução percentual das taxas de internações hospitalares por diarreias de cada tercil foram calculadas ao se dividir as reduções percentuais das taxas de hospitalizações por diarreia do segundo e terceiro tercil pelo primeiro tercil. Para a comparação estatística do segundo e terceiro tercis com o primeiro tercil utilizou-se teste não paramétrico de Kruskal-Wallis.

Em todas as etapas do estudo foi utilizado para a análise estatística o programa R-Project (versão 2.9.2) ${ }^{22}$.

Por se tratar de um estudo que utiliza bases de dados secundários, em acordo com a Resolução 196/96 o presente estudo não foi submetido à apreciação do Comitê de Ética para avaliação quanto aos riscos a seres humanos.

\section{Resultados}

Foram estudados 1.329 .618 nascidos vivos com menos de um ano de vida nos anos de $1999 \mathrm{e}$ 2008 em 25 capitais brasileiras e Distrito Federal. Em relação ao total de internações hospitalares registradas no SIH em 1999, o percentual de internações por diarreia diminuiu pela metade, re presentando $2,67 \%$ do total de internações em 2008 (Tabela 1).

As taxas de internações hospitalares por diarreia também reduziram significativamente entre 1999 e 2008, sendo que a redução da taxa de internações hospitalares por diarreia parece ter sido maior para os meninos que para as meninas (Tabela 2).

Tabela 1. Descrição da população de menores de um ano de vida, das internações hospitalares entre menores de um ano de vida em 25 capitais Brasileiras e Distrito Federal nos anos de 1999 e 2008.

\begin{tabular}{lrr}
\hline \multirow{2}{*}{ Variável } & \multicolumn{2}{c}{ Ano } \\
\cline { 2 - 3 } & \multicolumn{1}{c}{1999} & \multicolumn{1}{c}{2008} \\
\hline População de nascidos vivos & 714.745 & 614.873 \\
M eninos & 365.563 & 315.026 \\
Meninas & 347.015 & 299.763 \\
Ignorados & 2.167 & 84 \\
Internações hospitalares & 143.239 & 127.653 \\
M eninos & 80.586 & 69.731 \\
M eninas & 62.653 & 57.922 \\
Internações por diarreia & 7.759 & 3.508 \\
M eninos & 4.418 & 1.995 \\
Meninas & 3.341 & 1.513 \\
\hline
\end{tabular}

Tabela 2. Descrição das internações hospitalares em 25 capitais Brasileiras e Distrito Federal nos anos de 1999 e 2008.

\begin{tabular}{|c|c|c|c|c|}
\hline \multirow[t]{2}{*}{ Variável } & \multicolumn{2}{|c|}{ Ano } & \multirow{2}{*}{$\begin{array}{l}\text { Diferença } \\
\text { (1999-2008) }\end{array}$} & \multirow{2}{*}{$\begin{array}{l}\text { p-valor } \\
\text { (Wilcoxon) }\end{array}$} \\
\hline & 1999 & 2008 & & \\
\hline Total de internações hospitalares & $20,76 \%$ & $20,04 \%$ & $-0,72 \%$ & 0,093 \\
\hline Meninos & $22,04 \%$ & $22,13 \%$ & $0,09 \%$ & 0,048 \\
\hline Meninas & $18,05 \%$ & $19,32 \%$ & $1,27 \%$ & 0,135 \\
\hline $\begin{array}{l}\text { Internações por diarreia em relação ao total de } \\
\text { internações hospitalares }\end{array}$ & $5,41 \%$ & $2,74 \%$ & $-2,67 \%$ & 0,051 \\
\hline Meninos & $5,48 \%$ & $2,86 \%$ & $-2,62 \%$ & 0,054 \\
\hline Meninas & $5,33 \%$ & $2,61 \%$ & $-2,72 \%$ & 0,040 \\
\hline Taxa de internações hospitalares por diarreia (DNV)* & 10,86 & 7,04 & $-3,81$ & 0,035 \\
\hline Meninos* & 12,09 & 6,93 & $-5,75$ & 0,030 \\
\hline M eninas* & 9,63 & 5,05 & $-4,58$ & 0,030 \\
\hline
\end{tabular}

${ }^{*}$ Por mil nascidos vivos 
Os meninos são internados por diarreias numa taxa um quarto maior que as meninas, e essa diferença manteve-se relativamente constante entre 1999 e 2008. Já em relação ao total de internações hospitalares, parece haver uma proporção equivalenteentremeninos emeninas ( $\mathrm{Ta}$ bela 3).

0 aumento da prevalência de aleitamento materno exclusivo entre crianças com menos de quatro meses de vida teve correlação moderada com a redução das internações por diarreias, em ambos os sexos (Tabela 4).

Já a prevalência do al eitamento materno entre crianças com 9 a 12 meses de vida está fracamenteassociada, e marginal mentesignificante, à redução das internações hospitalares por diarreia, sendo essa correlação mais forte entre as meninas (Tabela 4).
Considerando os tercis de aumento da prevalência de al eitamento materno exclusivo, as cidades do primeiro tercil apresentaram taxas de hospitalização por diarreia em 1999 e 2008 inferiores as encontradas no segundo e terceiro tercis. No entanto, tanto as reduções absolutas quanto as reduções percentuais das taxas de internação foram maiores para o segundo e terceiro tercis que para o primeiro: o terceiro tercil experimentou uma redução percentual duas vezes maior nas taxas de internação que o primeiro, sendo essa redução estatisticamente significativa (Tabela 5).

\section{Discussão}

As taxas de internação hospitalar por diarreia diminuíram quase pela metadeentre 1999 e 2008,

Tabela 3. Descrição das razões de sexo das taxas de internação hospitalar, em 25 capitais Brasileiras e Distrito Federal nos anos de 1999 e 2008.

\begin{tabular}{|c|c|c|c|c|}
\hline \multirow{2}{*}{ Variável } & \multicolumn{2}{|c|}{ Ano } & \multirow{2}{*}{$\begin{array}{c}\text { Diferença } \\
\text { (1999-2008) }\end{array}$} & \multirow{2}{*}{$\begin{array}{c}\text { p-valor } \\
\text { (Wilcoxon) }\end{array}$} \\
\hline & 1999 & 2008 & & \\
\hline Razão de taxas de sexo do total de internações hospitalares** & 1,028 & 1,095 & $-0,07$ & 0,069 \\
\hline Razão de taxas de sexo das internações por diarreia (DNV) & 1,278 & 1,255 & $-0,01$ & 0,264 \\
\hline
\end{tabular}

${ }^{* *}$ Razão entremeninos/meninas

Tabela 4. Correlação entre as diferenças de prevalência de aleitamento materno e as diferenças das taxas de internações por diarreia entre menores de um ano de vida, entre os anos de 1999 e 2008.

\begin{tabular}{llc}
\hline \multicolumn{1}{c}{ Taxa de internações por diarreia } & Rho & p-valor \\
\hline Aleitamento M aterno Exclusivo & & 0,014 \\
Em relação ao total de crianças menores de um ano & $-0,483$ & 0,016 \\
$\quad$ M eninos & $-0,476$ & 0,008 \\
M eninas & $-0,521$ & 0,165 \\
Aleitamento materno das crianças de 9 a 12 meses de vida & & 0,250 \\
Em relação ao total de crianças menores de um ano & $-0,342$ & 0,119 \\
M eninos & $-0,286$ & $-0,381$ \\
M eninas
\end{tabular}

Tabela 5. Tercis de incremento da prevalência de aleitamento materno entre 1999 e 2008 e as taxas de internações hospitalares por diarreias.

\begin{tabular}{lccccc}
\hline $\begin{array}{c}\text { Incremento da prevalência } \\
\text { de aleitamento materno } \\
\text { exclusivo (1999 a 2008) }\end{array}$ & $\begin{array}{c}\text { Taxa em } \\
1999\end{array}$ & $\begin{array}{c}\text { Taxa em } \\
2008\end{array}$ & $\begin{array}{c}\text { Redução } \\
\text { absoluta } \\
\text { da Taxa }\end{array}$ & $\begin{array}{c}\text { Redução } \\
\text { percentual } \\
\text { da Taxa }\end{array}$ & $\begin{array}{c}\text { Razão de redução } \\
\text { percentual das } \\
\text { Taxas * }\end{array}$ \\
\hline $1^{\circ}$ tercil & 0,79 & 0,58 & 0,21 & $26,8 \%$ & 1,00 \\
$2^{\circ}$ tercil & 1,48 & 0,77 & 0,71 & $47,7 \%$ & 1,78 \\
$3^{\circ}$ tercil & 1,03 & 0,46 & 0,57 & $55,5 \%$ & 2,07 \\
\hline
\end{tabular}

* Teste de Kruskal-Wallis, valor de $p=0,042$ 
reduzindo, também pela metade, sua participação no total de internações ocorridas. 0 total de internações hospitalares, contudo, sofreu pequenas variações no período estudado.

Os objetivos deste estudo foram alcançados, pois verificou-se que o aumento da prevalência de crianças com menos de quatro meses de vida amamentadas exclusivamente esteve associado à diminuição das taxas de internação hospitalar por diarreias entre crianças com menos de um ano de vida entre 1999 e 2008. 0 aleitamento materno exclusivo esteve mais fortemente associado à redução das hospitalizações por diarreias entre as meninas.

As capitais que obtiveram maiores aumentos de prevalência de al eitamento materno exclusivo entre crianças com menos de quatro meses de vida, reduziram cerca de duas vezes mais seus percentuais de taxas de internação hospitalar por diarreia do que as capitais com menores aumentos daprevalência dealeitamento materno exclusivo.

Por outro lado, 0 aumento das prevalências de aleitamento materno entre crianças com 9 a 12 meses de vida parece ter influenciado menos as taxas de hospitalização por diarreias.

As diarreias podem ser responsáveis por 35\% a $86 \%$ das mortes infantis evitáveis, sendo que o aleitamento materno pode ser responsável pela redução de 9,3\% do Coeficiente de M ortalidade Infantil ${ }^{1}$. Um estudo realizado em M inas Gerais identificou uma queda de $50 \%$ da mortalidade por diarreias em 15 anos de série histórica, mas as diarreias, as pneumonias e a desnutrição permaneciam responsáveis por $16,5 \%$ das mortes ao final do período ${ }^{23}$, evidenciando que as diarreias permanecem sendo um importanteproblema de saúde pública.

A redução das internações hospitalares observadas nesse estudo por diarreia também foi observada em outros estudos brasileiros ${ }^{3,4}$. Benício e $M$ onteiro ${ }^{4}$, num inquérito conduzido na Cidade de São Paulo em 1984/85 e 1995/96, verificaram que a incidência anual de internações hospitalares reduziu de 2,21 para 0,79 internações por 100 crianças por ano.

Numa série de estudos de coorte realizados em Pelotas/RS, Matijasevich et al. ${ }^{3}$ verificaram uma redução estatisticamente significante das hospitalizações por diarreia (em relação ao total de internações) de 6,5\% em 1982, para 3,2 em 1993 e $1 \%$ em 2004.

Este estudo observou uma pequena, mas estatisticamente significativa, modificação do percentual do total de internações hospitalares. Já no estudo deM atijasevich et al. ${ }^{3}$ observou-seque o total de internações hospitalares não variou em 23 anos de acompanhamento.

U ma meta-análise de cinco estudos de países em desenvolvimento conduzida pela $\mathrm{OM} \mathrm{S}^{10}$ identificou que nos primeiros seis meses de vida, o al eitamento materno pode reduzir a mortalidade por diarreias (pooled OR $=6,1 ; \mathrm{IC} 95 \%=$ 4,1-9,0). Esse efeito protetor reduz, mas permanece importante, para crianças entre seis e onze meses (pooled OR = 1,9; IC95\% = 1,2-3,1).

0 aleitamento materno exclusivo parece reduzir, também, as hospitalizações por doenças diarreicas. Resultados com a mesma direção de associação foram encontrados em estudos observacionais $5^{6,11,12}$ e ecológicos ${ }^{13}$.

Uma coorte britânica com 15.890 crianças identificou que $o$ al eitamento materno exclusivo e o aleitamento materno podem reduzir em 53\% e 31\% a incidência de hospitalizações por diarreia (respectivamente) em crianças com menos de oito meses de vida6.

Um estudo do tipo caso-controle conduzido nas Filipinas identificou chance 10,5 vezes maior de hospitalização por diarreias entre crianças não amamentadas, comparadas as amamentadas exclusivamente ${ }^{11}$. A mesma chance foi observada num estudo multicêntrico da OM S conduzido em cinco países em desenvolvimento $(O R=10,5)$ com 9424 crianças. Porém, as populações comparadas foram crianças predominantemente amamentadas com as crianças não amamentadas ${ }^{24}$.

Num inquérito conduzido na cidade de Feira de Santana/BA, Vieira et al. ${ }^{12}$ verificaram que as crianças que não eram amamentadas ao seio exclusivamentetinham chance $82 \%$ maior deocorrência de diarreia.

Estudo ecológico conduzido por Boccolini e Boccolini ${ }^{13}$ verificou que a prevalência de al eitamento materno exclusivo pode reduzir em $25 \%$ as taxas de internação por doenças diarreicas de uma população.

O leite humano pode contribuir para a redução da incidência e severidade das doenças diarreicas devido aos seus diversos componentesimunológicos ${ }^{15}$ que se adaptam para atender às necessidades específicas de cada criança ${ }^{16}$, como os oligossacarídios que favorecem o crescimento de biffidobactérias no intestino ${ }^{19}$, impedindo a fixação deagentes patogênicosna mucosa intestinal ${ }^{20}$.

Um importante componente encontrado no leite humano para impedir infecções na mucosa intestinal, como as gastroenterites, é a proteína I gA-secretória ${ }^{17}$, uma vez que ela recobre todo 0 trato gastrointestinal da criança al imentada com leite materno ${ }^{18}$. 
A correlação entrea prevalência dealeitamento materno em crianças com menos de um ano de vida e as hospitalizações por diarreias foi fraca. Isso pode ter ocorrido pelo número reduzido decidades avaliadas nos anos de 1999 e 2008 ( $n=$ 18) e pelo fato do aleitamento materno com alimentação complementar ter um efeito protetor de menor magnitude que o do aleitamento materno exclusivo $0^{6,10}$. Contudo, esse efeito foi marginalmentesignificativo $(p<0,20)$.

A taxa de internação na população masculina foi maior que na população feminina, como 0 encontrado em outro estudo ${ }^{5}$. Como 0 aleitamento materno foi mais prevalente entre as meninas na pesquisa do Ministério da Saúde 7 , as mesmas podem ter menores taxas de internação por estarem mais protegidas deinfecções intestinais, conferida pelos componentes imunológicos dessa alimentação ${ }^{15-20}$.

Uma razão de taxa maior para os meninos também foi encontrada em outros estudos que observaram internações por doenças respiratórias $^{25,26}$, sendo que em uma coorte foi constatado que o efeito do aleitamento materno na redução das doenças respiratórias é maior entre as meninas que entre os meninos ${ }^{26}$.

$N$ ão ficou esclarecida a relação entreamamentação e gênero, e o porquê dos meninos adoecerem mais do que as meninas: por um lado os meninos tendem a ter mais problemas de saúde na infância, a permanecer mais tempo hospitalizados e a apresentar uma proporção maior de prematuridade do que as meninas ${ }^{27}$. Por outro lado, as meninas amamentam por mais tempo no Brasil ${ }^{7}$. Vale a pena ressaltar que a análise por sexo é importante porque além de enriquecer a descrição dos dados, permite quese levante pistas para pensar em motivos pelos quais diferenças por sexo desde tão tenra idade, pareçam influenciar a ocorrência de outros desfechos, como internação, amamentação e mortalidade infantil. É importante o desenvolvimento futuro de estudos qualitativos e quantitativos para esclarecimento das questões de gênero relacionadas aos padrões alimentares e de adoecimento na infância.

Outra limitação deste estudo é a não disponibilidade, até a data de publicação deste artigo, de dados secundários sobre a evolução das condições sanitárias e econômicas do período estudado, pois parte da redução observada neste estudo podeser devido à melhor cobertura da rede de distribuição de água e do aumento do poder aquisitivo da população ${ }^{4}$. Um estudo ecológico verificou que a proporção de pessoas com rede de abastecimento de água e a proporção de pessoas alfabetizadas em Capitais Brasileiras também influenciam na taxa média de internação H ospitalar ${ }^{13}$. Victora et al. ${ }^{14}$ relacionam a melhoria dos padrões estruturais e sociais brasileiros à redução da mortalidade infantil.

É interessante observar que o presente estudo, mesmo utilizando uma base de dados secundária sujeita a diversos vieses e quenão consiga contemplar todos os fatores de risco para as doenças diarreicas ${ }^{13}$, teve resultados semelhantes a diversos estudos observacionais, como: a relativa manutenção do total das internações hospitalares ${ }^{3}$; a redução das internações por diarreia $a^{3,4}$; uma razão de taxa de internações maior para os meninos ${ }^{25,26}$; e 0 efeito do aleitamento materno exclusivo na redução de hospitalizações por diarreias ${ }^{6,11,12}$.

I sso ressalta uma questão importante: a utilização de uma base de dados secundária, com ferramentas estatísticas e epidemiológicas adequadas, pode gerar inferências consistentes, e de uma forma pouco custosa, dos efeitos que as políticas públicas adotadas no país podem ter sobre a saúde da população.

Enfim, pode-se concluir que as cidades que conseguiram melhorar os padrões de aleitamento materno tiveram menores taxas de internação hospitalar por diarreias na população de crianças com menos de um ano de vida, reforçando a importância das políticas públicas de promoção, proteção e apoio ao aleitamento materno para o fortalecimento do Sistemaú nico deSaúdeepara a promoção da saúde.

\section{Colaboradores}

CS Boccolini, PMM Boccolini, ML Carvalho e MIC Olivei ra participaram igualmente de todas as etapas de elaboração do artigo. 


\section{Referências}

1. Escuder MML, Venâncio SI, Pereira JCR. Estimativa de impacto da amamentação sobre a mortalidade infantil. Rev Saude Publica 2003; 37(3):319-325.

2. World Health Organization (WHO). Medical education: teaching medical students about diarrhoeal diseases. Geneva: WHO; 1999.

3. Matijasevich A, Cesar JA, Santos IS, Barros AJD, Dode M ASO, Barros FC, Victora CG. Internações hospitalares durante a infância em três estudos de base populacional no Sul do Brasil: tendências e diferenciais. Cad Saude Publica 2008; 24(Supl. 3): S437-S443.

4. Benicio M HD'A, Monteiro CA. Tendência secular da doença diarreica na infância na cidade de São Paulo (1984-1996). Rev Saude Publica 2000; 34(6 Supl.):83-90.

5. Bittencourt SA, Leal MC, Santos MO. Hospitalizações por diarréia infecciosa no Estado do Rio de Janeiro. Cad Saude Publica 2002; 18(3):747-754.

6. Quigley MA, Kelly YJ, Sacker A. Breastfeeding and hospitalization for diarrheal and respiratory infection in the United Kingdom Millennium Cohort Study. Pediatrics 2007; 119(4):837-842.

7. Brasil. M inistério da Saúde (MS). Secretaria de Atenção à Saúde. Departamento de Ações Programáticas e Estratégicas. II Pesquisa de Prevalência de Aleitamento M aterno nas Capitais Brasileiras e Distrito Federal. Brasília: M S; 2009

8. Kramer MS, Kakuma R. World Health Organization: The optimal duration of breastfeeding - a systematic review. Geneva: WHO; 2002.

9. Kramer MS, Kakuma R. The optimal duration of exclusive breastfeeding: a systematic review. Adv Exp M ed Biol 2004; 554:63-77.

10. World Health Organization. Effect of breastfeeding on infant and child mortality due to infectious diseases in less developed countries: a pooled analysis. WHO Collaborative Study Team on the Role of Breastfeeding on the Prevention of Infant M ortality. Lancet 2000; 355(9202):451-455.

11. Hengstermann S, Mantaring JB 3rd, Sobel HL, Borja VE, Basilio J, Iellamo AD, Nyunt-U S. Formula feeding is associated with increased hospital admissions due to infections among infants younger than 6 months in Manila, Philippines. J Hum Lact 2010; 26(1):19-25.

12. Vieira GO, Silva LR, Vieira TO. Alimentação infantil e morbidade por diarréia. J Pediat 2003; 79(5):449454.

13. Boccolini CS, Boccolini PMM. Relação entre aleitamento materno e internações por doenças diarreicas nas crianças com menos de um ano de vida nas Capitais Brasileiras e Distrito Federal, 2008. Epidemiol. Serv. Saúde 2011; 20(1):19-26.

14. Victora CG, Aquino EM, do Carmo Leal M, Monteiro CA, Barros FC, Szwarcwald CL. M aternal and child health in Brazil: progress and challenges. Lancet 2011; 377(9780):1863-1876.
15. Paramasivam K, Michie C, Opara E, Jewell AP. Human breast milk immunology: a review. Int J Fertility Women's M ed 2006; 51(5):208-217.

16. Goldman AS, Garza C, Nichols BL, Goldblum RM Immunologic factors in human milk during the first year of lactation. J Pediatrics 1982; 100(4):563-567.

17. Cruz JR, Gil L, Cano F, Caceres P, Pareja G. Breast milk anti-Escherichia coli heat-labile toxin IgA antibodies protect against toxin-induced infantile diarrhea. Acta Paediatrica Scandinavica 1988; 77(5):658662.

18. Davidson LA, Bo L. Persistence of human milk proteins in the breast-fed infant. Acta Pediatrica 1987; 76(5):733-740.

19. Newburg DS. Neonatal protection by an innate immune system of human milk consisting of oligosaccharides and glycans. J Anim Sci 2009; 87(Supl. 13): :26-34.

20. M orrow $A L$, Ruiz-Palacios $G M$, Jiang $X, N$ ewburg DS. H uman-M ilk glycans that inhibit pathogen binding protect breast-feeding infants against infectious diarrhea. J Nutr. 2005; 135(5):1304-1307.

21. Brasil. M inistério da Saúde. Informações de saúde [acessado 2011 abr 1]. Disponível em http://www. datasus.gov.br

22. The R Foundation for Statistical Computing. [página na Internet]. [acessado 2010 jan 5]. Disponível em: http://www.r-project.org

23. Caldeira AP, França E, Perpétuo IHO, Goulart EMA. Evolução da mortalidade infantil por causas evitáveis, Belo Horizonte, 1984-1998. Rev Saude Publica 2005; 39(1):67-74.

24. Bahl R, Frost C, Kirkwood BR, Edmond K, Martines J, Bhandari N, Arthur P. Infant feeding patterns and risks of death and hospitalization in the first half of infancy: multicentre cohort study. Bull World Health Organ 2005; 83(6):418-426.

25. Macedo SEC, M enezes AM B, Albernaz E, Post $P$, Knorst M. Fatores de risco para internação por doença respiratória aguda em crianças até um ano de idade. Rev Saude Publica 2007; 41(3):351-358.

26. Klein MI, Bergel E, Gibbons L, Coviello S, Bauer G, Benitez A, Serra ME, Delgado MF, Melendi GA, Rodríguez S, Kleeberger SR, Polack FP. Differential gender response to respiratory infections and to the protective effect of breast milk in preterm infants. Pediatrics 2008; 121(6):1510-1516.

27. Gissler $M$, Järvelin $M R$, Louhiala $P$, Hemminki $E$. Boys have more health problems in childhood than girls: follow-up of the 1987 Finnish birth cohort. Acta Paediatr 1999; 88(3):310-314.

Artigo apresentado em 07/04/2011

Aprovado em 07/08/2011

Versão final apresentada em 21/10/2011 